\title{
Risk Factors for Inpatient Hospital Admission in Pediatric Burn Patients
}

\author{
Alvin To ${ }^{1}$, Yana Puckett ${ }^{2}$ \\ 1. Saint Louis University School of Medicine 2. Department of Surgery, Texas Tech University Health \\ Sciences Center
}

$\square$ Corresponding author: Alvin To, toak@slu.edu

Disclosures can be found in Additional Information at the end of the article

\section{Abstract}

\section{Purpose}

Our objective was to determine the risk factors for inpatient admission of pediatric burn patients.

\section{Materials \& methods}

This cross-sectional study uses data from the Healthcare Cost and Utilization Project Kids' Inpatient Database (HCUP KID) for the years of 2003, 2006, 2009, and 2012 to estimate the risk factors for inpatient admission for pediatric patients who sustained a burn injury. Patients who sustained a burn between the ages of 1 and 18 years were included.

\section{Results}

A total of 43,453 patients met inclusion criteria. Of those, $42.3 \%$ were Caucasian, $20.1 \%$ were African American, and 19.3\% were Hispanic. Males comprised 63.5\% of the studied population. The month of July was associated with a $31.8 \%$ increased chance $(\mathrm{p}=.011)$ of being admitted to hospital for a pediatric burn. It was found that patients being admitted had a $32.2 \%$ increased chance $(p=.002)$ of a fluid and electrolyte abnormality and a $61.0 \%$ increased chance $(p=.027)$ of drug abuse.

\section{Conclusions}

Pediatric burn patients are more likely to be admitted to the hospital having a fluid and electrolyte abnormality, having a drug abuse status, and/or during the month of July.

Received 04/03/2016

Review began 04/05/2016 Review ended 04/24/2016 Published 05/06/2016

\section{(c) Copyright 2016}

To et al. This is an open access article distributed under the terms of the Creative Commons Attribution License CC-BY 3.0., which permits unrestricted use, distribution, and reproduction in any medium, provided the original author and source are credited.
Categories: Pediatrics, Epidemiology/Public Health

Keywords: burns, patient admission, pediatrics, periodicity, seasons, risk factors, epidemiology, public health

\section{Introduction}

In the United States, burn injuries affect a million children annually [1]. The cost of pediatric burn-associated hospitalizations and the total cost of burn-associated expenditure are estimated at around $\$ 211,772,700$ and $\$ 2.1$ billion, respectively [2-3]. Up to $91.3 \%$ of burn injuries are accidental; these cases may potentially be prevented by active strategies, such as educating at-risk populations on behavioral changes and environmental control, and passive strategies, such as installing safety devices [4]. Categorizing risk factors for burns may aid in 
devising burn prevention strategies.

Over $50 \%$ of pediatric burn injuries occur in children under the age of five with scald injuries being the major etiology [4]. Accidental burns are more likely to occur if the child is either in a dangerous environment or insufficiently supervised. As the safety of the environment and degree of supervision varies temporally, it follows that there may be a temporal pattern of burn injury presentation corresponding with the time of the day, day of the week, or month/season of the year. All-age studies around the world have presented discordant conclusions: some findings suggest increased prevalence of admission during summer [5-9], winter [10], both summer and winter [11-12], or both winter and spring [13-15]; some studies found no correlation with the seasons [16-18]. It is likely that population-specific factors, such as cultural norms, ethnicity, or local climate, may alter the behaviors of each study sample differently. Some of these behaviors may place individuals in environments that pose a greater risk for accidental burns, specific to certain months of the year. Within the United States, pediatric and all-age studies examining seasonal variation of burn risk have been limited in number and only represent a limited geographic range $[5,11,19]$; thus, there is a need to investigate admission patterns in the United States, which may guide prevention or burn treatment strategies.

In this study, we retrospectively examine a national pediatric database for burn injuries and inpatient admission. We assessed risk factors for admission including weekend status, month of admission, race, and payer status. Consent was obtained by all participants in this study.

\section{Materials And Methods}

Using data from the Healthcare Cost and Utilization Project Kids' Inpatient Database (HCUP KID) compiled for the years of 2003, 2006, 2009, and 2012, we performed a retrospective analysis of all pediatric burn patients. Patient-related variables obtained from the database included age (years), sex, race (Caucasian, African American, Hispanic, Asian or Pacific Islander, Native American, and other), in-hospital death, fluid and electrolyte disorder, and history of drug abuse. Each record contained discharge diagnosis and procedure codes defined by the 15th International Classification of Diseases, Ninth Revision, Clinical Manifestations (ICD-9-CM). ICD-9 codes included 940.0-949.5.

Comorbid conditions were identified on the basis of both the ICD-9 diagnosis and procedure codes and the Clinical Classification Software diagnosis and procedure classifications. Statistical Package for the Social Sciences (SPSS) (IBM Corporation, New York, USA) was utilized for data analysis. Logistic regression was utilized to evaluate effect of month of admission, weekend status, gender, race, fluid and electrolyte abnormalities, drug abuse, age, and procedures required for all pediatric burn injuries on admission from 2003 to 2012. Estimations with $\mathrm{P}$ values less than .05 were considered statistically significant.

\section{Results}

A total of 43,453 patients were analyzed. The average age of patients was $6.35(\mathrm{SD}=8.37)$, and $63.5 \%$ were male. Caucasians accounted for $42.3 \%$ of the population; African Americans, 20.1\%; and Hispanics, $19.3 \%$. Medicaid was the most common form of payment (51.3\%), followed by private insurance (34.4\%), and self-pay (6.9\%). A total of 203 patients with burns did not survive, and the average number of procedures on admission was 2.08 ( $\mathrm{SD}=3.1)$ (Table 1). 


\section{Cureus}

\section{Variable}

Age

Male gender

Mortality

Number of procedures on admission

Race

Caucasian

African American

Hispanic

Asian or Pacific Islander

Native American

Other

Primary expected payer

Medicare

Medicaid

Private Insurance including HMO

Self-pay

No charge

Other (title v, worker's compensation, CHAMPUS/CHAMPVA, other government)

Obesity

Fluid and electrolyte disorder
Mean (SD) Percent (N)

$6.35(8.37)$

$63.5 \%(27,310)$

$0.5 \%(203)$

$2.08(3.1)$
$42.3 \%(16,388)$

$20.1 \%(7771)$

$19.3 \%(7475)$

$2.5 \%(970)$

$0.9 \%(362)$

$7.5 \%(2902)$

$0.2 \%(79)$

$51.3 \%(22,272)$

$34.4 \%(14,930)$

$6.9 \%(2,991)$

$0.2 \%(69)$

$7.1 \%(3,065)$

$0.6 \%(255)$

$6.1 \%(2662)$

TABLE 1: Demographics of National Weighted Pediatric Burn Patients Across United States From 2003-2012

$(\mathrm{N}=43,453)$

The effects of month of admission, weekend status, gender, race, fluid and electrolyte abnormalities, drug abuse, age, and procedures required for all pediatric burn injuries on admission from 2003 to 2012 were evaluated by logistic regression. The results revealed that a pediatric burn patient was $31.8 \%$ more likely to be admitted to hospital in the month of July compared to other months $(\mathrm{p}=.011)$. The months of January and April were associated with decreased chances of admission by $44 \%$ and $13 \%$, respectively. 


\section{Cureus}

African Americans and Hispanics pediatric burn patients were associated with decreased odds of admission by $15 \%$ and $30 \%$, respectively. Gender was not found to be statistically significant for odds of inpatient admission in pediatric burn patients. Patients had a two percent increased chance of being admitted to the hospital if a procedure was required $(\mathrm{p}=.001)$.

Children who were younger than five years old were less likely to be admitted by $25 \%(\mathrm{p}<.0001)$ compared to children older than five years of age. However, ages between six to ten and 11-15 were not statistically significant for either an over- or under-representation of inpatient admission.

Fluid and electrolyte abnormalities and drug abuse were factors associated with need for admission to hospital by $32.3 \%$ and $61 \%$, respectively. Private insurance payment type was associated with a decreased chance for inpatient admission by $30 \%(\mathrm{p}<.0001)$ (Table 2$)$.

Variable

Age (years)

$0-5$

6-10

$11-15$

Sex (female)

Procedures required on admission

Mortality

Weekend admission

Month of admission

January

$.563(.366-.866)$

.868 (.683-1.104)

1.318 (1.065-1.632)

July

Race

Caucasian

$1.190(.964-1.469)$

$.850(.720-1.003)$

$.700(.582-.842)$

Hispanic

Payment type

Medicare

$.000(.000-.000)$

Medicaid

$1.252(.616-2.543)$

.696 (.609-.794)

$.986(.862-1.127)$

p

.000

.164

.164

.751

.001

.008

.000

.009

.003

.011

Private insurance including $\mathrm{HMO}$

Self-Pay
.999

.105

.054

.000

.534

.000

.831 


\section{Cureus}

No Charge

Other (title v, worker's compensation, CHAMPUS/CHAMPVA, other government)

Obesity

Fluid and electrolyte abnormality

Drug abuse
$.774(.644-.924)$

.006

$.472(.138-1.616)$

$1.407(.792-2.500)$

.244

$1.322(1.105-1.581)$

.002

$1.610(1.056-2.454)$

.027

\section{TABLE 2: Logistic Regression Models}

Effect of Month of Admission, Weekend, Gender, Race, Fluid and Electrolyte Abnormalities, Drug Abuse, and Age, Procedures Required for Pediatric Burn Injuries on Admission from 2003-2012 (N=28, 905).

\section{Discussion}

In this study, pediatric-burn associated hospitalizations were examined from a national sample of cases using HCUP KID data. Current findings on the seasonal distribution of pediatric burn admissions have been limited in sample size and geographic scope, and as a result, present discordant conclusions. Our study is based on a national dataset and suggests a $31.8 \%$ increased likelihood for a burn-associated pediatric patient to be admitted during July. Furthermore, to our knowledge, this study is the first to examine a national burn injury population and establishes a correlation between odds of admission with fluid and electrolyte abnormalities and/or drug abuse status.

In this analysis, patients identifying as African American and Hispanic comprise $20.1 \%$ and $19.3 \%$ of the pediatric burn cases, respectively. In contrast, census data indicates that African Americans and Hispanics comprise $12.6 \%$ and $16.3 \%$ of the general population, respectively [20]. These demographic groups are overrepresented in pediatric burn cases within the United States-a finding also in line with previous research [4].

Interestingly, our analysis showed that despite over-representing the pediatric burn population, African Americans and Hispanics had 15\% and 30\% decreased odds, respectively, of being admitted compared to Caucasians. The decreased odds of admissions may be explained by an increase in non-urgent cases in these demographics. It has been suggested that the Emergency Medical Treatment and Active Labor Act (EMTALA) has increased the incidence of emergency department usage by uninsured patients who have non-urgent medical injuries [2122]. Nationally $11.8 \%$ and $19.9 \%$ of African Americans and Hispanics are uninsured, respectively; in comparison, $10.1 \%$ of Caucasians are uninsured [23]. Increased visits by patients with non-urgent needs could elevate the number of reported burn injuries. These minor burn injuries may be treated in the emergency department without need for hospital admission, thus lowering the apparent odds of admission.

Previous studies investigating temporal variations of burn inpatient admissions in the United States have had limited sample sizes representing a few states [5, 11, 19]; these studies' conclusions have not been congruent and can only be applied to the state where each study was conducted. Our analysis represents pediatric burn patients nationally and shows a $31.8 \%$ increased likelihood for patients to be admitted during the month of July. Some smaller studies have demonstrated higher rates of hospital admissions during winter months, correlating with 
increased scald injuries possibly caused by consumption of hot beverages and use of electric heaters [11]. Our data, however, does not suggest a national increase of burn injury admissions during winter months.

Our observation of an increased risk of admission during July may represent risks that are more general to the national population. The Fourth of July holiday, celebrated with fireworks, may pose a significant burn risk. Annually, around 10,000 firework-related injuries occur in the United States [24]. Furthermore, 60\% of firework injuries involve pediatric populations predominantly affecting 10-14 year old males [25]. A study found that pediatric firework injuries are 5.94 times more likely to occur within five days around Fourth of July compared to other holidays [26]. It should be noted, however, that $91.6 \%$ of children who receive emergency treatment for firework-related injuries are not admitted. Therefore, Fourth of July related firework burns might not sufficiently account for the increase in pediatric burn admissions in July. Increases in temperature around summer may be an additional contributor. Small sample studies have documented that infants and preschoolers are at risk of plantar burns caused by sun-heated surfaces at home and in public play areas [27-28].

Pediatric burn patients who presented with a history of drug abuse (amphetamines, barbiturates, opioids, and/or cannabinoids) were more likely to be admitted by $61 \%(\mathrm{p}=.027)$. Harmful behaviors associated with substance abuse increases the risk of adverse burn outcomes $[19,29]$. Up to $60.6 \%$ of incidents reported in these studies involved alcohol consumption; these patients had increased surface area of burn coverage, greater number of medical procedures, and lengthened hospitalizations. Within our study, burn patients presenting with a history of drug abuse may have been admitted to treat not only their burns, but also their substance abuse, which may explain the increased inpatient admission rate within this demographic.

Some limitations of this study need to be recognized. The study population represents individuals seeking emergency department care, including those who were subsequently admitted. Outpatient burn victim data is not recorded in HCUP KID. Minor burn cases may be significant, although underrepresented, in this study due to the possibility of outpatient selfmedication. Therefore, the correlations observed in our study may be more applicable to moderate or severe burn cases presenting in the Emergency Department.

\section{Conclusions}

This study reports a correlation between national pediatric burn admissions and a history of drug abuse and/or fluid and electrolyte abnormalities within the United States. Nationally, the risk for children to be admitted for burns is greatest during the month of July. These findings may assist the development of future strategies aimed at reducing pediatric burns.

\section{Additional Information}

\section{Disclosures}

Human subjects: Consent was obtained by all participants in this study. Animal subjects: All authors have confirmed that this study did not involve animal subjects or tissue. Conflicts of interest: In compliance with the ICMJE uniform disclosure form, all authors declare the following: Payment/services info: All authors have declared that no financial support was received from any organization for the submitted work. Financial relationships: All authors have declared that they have no financial relationships at present or within the previous three years with any organizations that might have an interest in the submitted work. Other relationships: All authors have declared that there are no other relationships or activities that could appear to have influenced the submitted work. 


\section{References}

1. Gonzalez R, Shanti CM: Overview of current pediatric burn care. Semin Pediatr Surg. 2015, 24:47-49.

2. Shields BJ, Comstock RD, Fernandez SA, et al.: Healthcare resource utilization and epidemiology of pediatric burn-associated hospitalizations, United States, 2000. J Burn Care Res. 2007, 28:811-826. 10.1097/BCR.0b013e3181599b51

3. Miller TR, Finkelstein AE, Zaloshnja E, et al.: Injury Prevention for Children and Adolescents: Research, Practice, and Advocacy. Liller KD (ed): American Public Health Association, Washington DC; 2006.

4. Kramer CB, Rivara FP, Klein MB: Variations in U.S. pediatric burn injury hospitalizations using the national burn repository data. J Burn Care Res. 2010, 31:734-739.

10.1097/BCR.0b013e3181eebe76

5. Stuke LE, Arnoldo BD, Hunt JL, et al.: Hydrofluoric acid burns: a 15-year experience. J Burn Care Res. 2008, 29:893-896. 10.1097/BCR.0b013e31818b9de6

6. Vermaak P, Haj Basheer M, Taki H, et al.: Paediatric injuries associated with the use of disposable barbecues: are we finding ourselves in hot sand?. Burns. 2012, 38:763-766. 10.1016/j.burns.2011.12.009

7. Yao Y, Liu Y, Zhou J, et al.: The epidemiology of civilian inpatients' burns in Chinese military hospitals, 2001-2007. Burns. 2011, 37:1023-1032. 10.1016/j.burns.2011.03.021

8. Rainey S, Cruse CW, Smith JS, et al.: The occurrence and seasonal variation of accelerantrelated burn injuries in central Florida. J Burn Care Res. 2007, 28:675-680. 10.1097/BCR.0b013E318148C86E

9. Han TH, Kim JH, Yang MS, et al.: A retrospective analysis of 19,157 burns patients: 18 -year experience from Hallym Burn Center in Seoul, Korea. Burns. 2005, 31:465-470. 10.1016/j.burns.2004.11.014

10. Lin TM, Wang KH, Lai CS, et al.: Epidemiology of pediatric burn in southern Taiwan . Burns. 2005, 31:182-187. 10.1016/j.burns.2004.07.015

11. Hultman CS, Tong WT, Surrusco M, et al.: To everything there is a season: impact of seasonal change on admissions, acuity of injury, length of stay, throughput, and charges at an accredited, regional burn center. Ann Plast Surg. 2012, 69:30-34. 10.1097/SAP.0b013e31823f3df0

12. $\mathrm{Xu} \mathrm{JH}$, Qiu J, Zhou JH, et al.: Pediatric burns in military hospitals of China from 2001 to 2007: a retrospective study. Burns. 2014, 40:1780-1788. 10.1016/j.burns.2014.04.003

13. Chen XJ, Sun WJ, Wang J, et al.: Epidemiology of bedside stove burns in a retrospective cohort of 5089 pediatric patients. Burns. 2014, 40:1761-1769. 10.1016/j.burns.2014.03.018

14. Frans FA, Keli SO, Maduro AE: The epidemiology of burns in a medical center in the Caribbean. Burns. 2008, 34:1142-1148. 10.1016/j.burns.2008.05.013

15. Hemeda M, Maher A, Mabrouk A: Epidemiology of burns admitted to Ain Shams University Burns Unit, Cairo, Egypt. Burns. 2003, 29:353-358. 10.1016/S0305-4179(03)00044-5

16. Verey F, Lyttle MD, Lawson Z, et al.: When do children get burnt?. Burns. 2014, 40:1322-1328. 10.1016/j.burns.2014.01.010

17. Pan YC, Huang HM, Chen F, et al.: Epidemiological investigation of burn inpatients in 6 hospitals of Hainan province: a retrospective analysis in 8 years. Zhonghua Shao Shang Za Zhi. 2011, 27:371-374.

18. Ho WS, Ying SY: An epidemiological study of 1063 hospitalized burn patients in a tertiary burns centre in Hong Kong. Burns. 2001, 27:119-123. 10.1016/S0305-4179(00)00095-4

19. Fraga AM, Fraga GP, Noordenbos J, et al.: Beach and campfire burns: a site of pleasure and tragedy. J Burn Care Res. 2010, 31:184-189. 10.1097/BCR.0b013e3181c7ed46

20. Overview of race and Hispanic origin: 2010 . (2011). Accessed: December 22, 2015 : http://www.census.gov/prod/cen2010/briefs/c2010br-02.pdf.

21. Twanmoh JR, Cunningham GP: When overcrowding paralyzes an emergency department. Manag Care. 2006, 15:54-59.

22. Honigman LS, Wiler JL, Rooks S, et al.: National study of non-urgent emergency department visits and associated resource utilization. West J Emerg Med. 2013, 14:609-616. 10.5811/westjem.2013.5.16112

23. Health insurance coverage in the United States: 2014 . (2015). Accessed: December 22, 2015: https://www.census.gov/content/dam/Census/library/publications/2015/demo/p60-253.pdf. 


\section{Cureus}

24. See LC, Lo SK: Epidemiology of fireworks injuries: the National Electronic Injury Surveillance System, 1980-1989. Ann Emerg Med. 1994, 24:46-50. 10.1016/S0196-0644(94)70161-X

25. Witsaman RJ, Comstock RD, Smith GA: Pediatric fireworks-related injuries in the United States: 1990-2003. Pediatrics. 2006, 118:296-303. 10.1542/peds.2006-0790

26. D'Ippolito A, Collins CL, Comstock RD: Epidemiology of pediatric holiday-related injuries presenting to US emergency departments. Pediatrics. 2010, 125:931-937. 10.1542/peds.20090307

27. Asquith C, Kimble R, Stockton K: Too hot to trot (barefoot)... A study of burns in children caused by sun heated surfaces in Queensland, Australia. Burns. 2015, 41:177-180.

10.1016/j.burns.2014.06.011

28. Sinha M, Salness R, Foster KN, et al.: Accidental foot burns in children from contact with naturally heated surfaces during summer months: experience from a regional burn center. J Trauma. 2006, 61:975-978. 10.1097/01.ta.0000229810.36074.36

29. Neaman KC, Do VH, Olenzek EK, et al.: Outdoor recreational fires: a review of 329 adult and pediatric patients. J Burn Care Res. 2010, 31:926-930. 10.1097/BCR.0b013e3181f938f7 